Aus der Fakultat fur Landwirtschaft der Universilat Damaskus' und dem Institut fur Nutztierwissensehaften der Landwirtschnflich-Gärtnerischen Fakultat der Humboldi-Universitat zu Berliñ

BASSAM SALLAH ISSA' und GERHARD SEELAND?

\title{
Einfluss von Inzucht und Selektion auf die Fruchtbarkeit und das Wachstum der Maus
}

\author{
Herrn Prof. Dr. Dr. h. c. Hans, Joachim Schwark zum 75. Geburtstag gewidmet
}

\begin{abstract}
Summary
Title of the paper: Effect of inbreeding and selection on fertility and growth in mice

The experiment comprised 3 lines of outbred mice: An inbred line without selection (1), an inbred line with selection (2) and a non inbred control line with random mating (3). In line 1 and 2 half sister mating was used. In line $2,20 \%$ of the females are selected for litter size and stillbirths of their dam. The males are selected at two stages: $50 \%$ for body weight at the age of 10 days and $50 \%$ for body weight at the age of 21 days. Inbreeding depressions seems to depend non linear on the degree of inbreeding. Selection within the line 2 could compensate inbreeding depression for fertility and growth. Epistatic effects and natural selection are discussed as a cause of the non linear inbreeding depression.
\end{abstract}

Key Words; mice, inbreeding depression, selection, growth, fertility

\section{Zusammenfassung}

Das Experiment umfasste drei Linien ausgezuchteter Māuse: Eine Inzuchtlinie ohne Selektion (1), eine Inzuchtlínie mit Selektion (2) und eine nicht ingezuchtete Kontrolllinie. Die Vermehrung crfolgte in den Inzuchtlinien durch Halbgeschwisterpaarungen. In der Linie 2 wurden $20 \%$ der weiblichen Tiere nach der Mutterleistung (Wurfgröße und Anzahl der Totgeburten) sowie 25\% der männlichen Tiere nach dem Körpergewicht am 10. Lebenstag $(50 \%)$ und nach dem Korpergewicht am 21 . Lebenstag $(50 \%)$ selektiert. Die Inzuchtdepressionen verhielten sich nicht proportional zum Inzuchtgrad. Durch die Selektion war es möglich, die Inzuchtdepressionen in der Linie 2 zu kompensieren. Als Grinde fur den nichtlinearen Zusammenhang zwischen Inzuchtgrad und Inzuchtdepression werden epistatische Geneffekte und naturliche Selektion diskutiert.

Schl(usselwörter: Maus, Inzuchtdepression, Selektion, Wachstum, Fruchtbarkeit

I. Einleitung

In dem Bemühen, alle Möglichkeiten für die Steigerung des Zuchtfortschritts zu nutzen, wird die Inzucht als eine Maßnahme zur Erhöhung der genetischen Variabilität innerhalb der Population durch Linienbildung diskutiert (PIRCHNER u.a., 1989; RENNE u.a., 1997), wenn auch die Ergebnisse nicht eindeutig ausfallen (SMITH und QUINTON, 1993). Dazu müssen die Fragen nach dem Grad der Inzucht in den Populationen, der Eingliederung der Inzucht in die Zuchtprogramme und den Auswirkungen auf die phänotypischen Merkmale, den Inzuchtdepressionen, neu gestellt werden. Da die Höhe der Inzuchtdepressionen von vielen Faktoren, wie dem Merkmal, der Tierart, aber auch dem Paarungssystem und von der Selektion abhängig sind (DIETL u.a., 2001), können Verallgemeinerungen kaum vorgenommen werden. 
In der vorliegenden Arbeit wurde der Einfluss der Inzucht auf die Merkmale der Fruchtbarkeit und des Wachstums bei dem Modelltier Labormaus über 8 Generationen untersucht. Gleichzeitig wurde geprüf, inwieweit eine Selektion auf die Merkmale die Inzuchtdepression kompensieren kann.

2. Material und Methode

In das Experiment wurden 3 Linien von ursprünglich ausgezüchteten Mäusen einbezogen:

- eine Inzuchtlinie ohne Selektion (1)

- eine Inzuchtlinie mit Selektion (2)

- eine Kontrolllinie (3).

Der Versuch wurde mit 60 männlichen und 60 weiblichen Tieren pro Linie begonnen. Für die folgenden Generationen sah der Paarungsplan vor, das Verhältnis von männlichen zu weiblichen Tieren auf 1:2 zu erweitern, so dass jeweils 20 männliche und 40 weibliche Tiere je Linie fur die Weiterzucht auszuwählen waren. In den Linien 1 und 2 erfolgte die Auswahl der Eltern für die năchste Generation unter Berlicksichtigung des Verwandtschaftsgrades, um eine Halbgeschwisterpaarung zu erreichen. In der Linie 3 erfolgte Zufallspaarung. Der durchschnittliche Inzuchtanstieg in den ersten $8 \mathrm{Ge}$ nerationen ist für die Linien 1 und 2 in den Ergebnistabellen dargestellt. Die relativ enge Verwandtschaftsanpaarung führte zu einem schnellen Anstieg der Inzucht.

In der Linie 2 wurden die weiblichen Tiere nach der Fruchtbarkeit und die männlichen Tiere auf Wachstum selektiert. Die Selektion der männlichen Tiere erfolgte in 2 Stufen. in der 1 . Stufe wurde mit einer Intensität von $50 \%$ nach dem Körpergewicht im Alter von 10 Tagen selektiert. Die ausgewählten Tiere wurden im Alter von 21 Tagen abermals nach dem Körpergewicht selektiert. Da in der 2. Stufe die Selektionsintensität ebenfalls $50 \%$ betrug, ergibt sich eine Gesamtselektionsintensität für beide Stufen von $75 \%$. Die weiblichen Tiere der Linie 2 wurden nach den Leistungen der Mutter in den Merkmalen Wurfgröße bei der Geburt und Anzahl der Totgeburten im Wurf selektiert. Die Remontierungsrate betrug durchschnittlich $20 \%$.

Die Schätzung der Inzuchtdepression erfolgte uber eine lineare Regression der Inzuchtkoeffizienten auf die Merkmalswerte. Da die Inzuchtzunahme in Einheiten von $10 \%$ betrachtet wurde, sind die Inzuchtdepressionen auf dieses $\mathrm{Maß}$ bezogen worden.

$y=\alpha+b x$

$\mathrm{y}=$ Merkmalswert

$\alpha=$ Regressionskonstante

$\mathrm{b}=$ Regressionskoeffizient

$\mathrm{x}=$ Inzuchtgrad

Tabelle I

Zusammensetzung des verwendeten Alleinfutters im Experiment (Composition of the complete feed)

\begin{tabular}{lc}
\hline Komponente & Anteil (\%) \\
\hline Rohprotein & 22,18 \\
Rohfaser & 3,10 \\
Calcium & 1,20 \\
Phosphor & 0,58 \\
Lysin & 0,87 \\
Methionin & 0,54 \\
Trytophan & 0,25 \\
\hline
\end{tabular}


Den Tieren stand eine Alleinfuttermischung ad libitum zur Verfügung. Die Zusammensetzung der Futtermischung ist aus der Tabelle 1 ersichtlich.

\section{Ergebnisse}

In der Tabelle 2 sind die Generationsmittelwerte für das Merkmal Wurforöße bei Geburt der drei Linien dargestellt. Wie zu erwarten, wirkt sich der steigende Inzuchtgrad in der Linie 1 negativ auf das Fruchtbarkeitsmerkmal aus. Diese Tendenz ist in den absoluten Merkmalswerten bereits zu erkennen, kommt aber in den Abweichungen zur Kontrollgruppe noch deutlicher zum Ausdruck.

Tabelle 2

Mittelwerte der Wurfgroße bei der Geburt (WG 0) (Means of the litter size at birth)

\begin{tabular}{ccccc}
\hline Generation & $\mathrm{F}_{\mathrm{x}}$ & Linie 1 & Linie 2 & Linie 3 \\
\hline 0 & 0 & 11,20 & 12,60 & 10,90 \\
1 & 0,125 & 7,28 & 11,30 & 11,20 \\
2 & 0,218 & 8,03 & 10,20 & 11,00 \\
3 & 0,304 & 7,06 & 10,40 & 10,90 \\
4 & 0,380 & 6,09 & 9,70 & 10,50 \\
5 & 0,448 & 6,08 & 11,01 & 11,30 \\
6 & 0,508 & 7,01 & 11,07 & 11,10 \\
7 & 0,562 & 7,00 & 12,40 & 10,80 \\
8 & 0,610 & 7,02 & 12,40 & 9,70 \\
\hline
\end{tabular}

Wie die Ergebnisse in der Linie 2 zeigen, kann die Inzuchtdepression der Wurfgröße durch eine gleichzeitige Selektion auf dieses Merkmal weitgehend egalisiert werden. Eine annähernd vollständige Kompensation der Inzuchtdepression durch den Selektionserfolg gelingt jedoch erst gegen Ende des Versuches. Während der Generationen 2-4 müssen die größten Leistungseinbußen hingenommen werden. In der Inzuchtlinie ohne Selektion ist in der 3. und 4. Generation ein deutliches Tief der Merkmalswerte zu erkennen, während in den letzten Generationen die Wurfgröße wieder ansteigt. Der Einfluss der Inzucht auf das Merkmal ist signifikant, wie der entsprechende Regressionskoeffizient zeigt (s. Tab. 6).

Die Mittelwerte des Merkmals Anzahl der Totgeburten über die 8 Generationen sind in der Tabelle 3 dargestelit, Es kommt zu einem deutlichen Anstieg der Totgeburten von der 0. zur 1. Generation. Die höchste Anzahl Totgeburten sind in den Generationen 4 und 5 zu verzeichnen. Gegen Ende der Inzuchtphase nimmt sie wieder ab. Die Ursache könnte z.T. darin liegen, dass bei dem höheren Inzuchtgrad die Wurfgröße geringer ist und aus diesem Grunde die Anzahl der Totgeburten zurückgeht. Der lineare Regressionskoeffizient über alle Generationen als Maß für die Inzuchtdepression fällt negativ aus, ist aber nicht signifikant (vgl. Tab. 6). In der Kontrollgruppe liegen die Mittelwerte der Totgeburten uber alle Generationen auf einem erstaunlich einheitlichen Niveau.

Eine Selektion auf Fruchtbarkeit scheint sich auch günstig auf die Anzahl der Totgeburten auszuwirken, da in der Linie 2 in allen Generationen geringere Totgeburten auttreten als in der Linie 1.

Den Einfluss der Inzucht auf das Wachstum und die Kompensationsmoglichkeit durch Selektion geben die Tabellen 4 und 5 wieder. Wie zu erwarten, sind die Depressionen in den Wachstumsmerkmalen wegen der höheren Heritabilităt der Merkmale geringer als bei den Fruchtbarkeitsmerkmalen. Allerdings zeigt sich auch hier bei den beiden untersuchten Wachstumsmerkmalen, dass die höchsten Depressionen in den mittleren 
Generationen zu finden sind und nicht in der 8. Generation am Ende des Versuches, wo der höchste Inzuchtgrad erreicht wird. Eine Kompensation der Inzuchtdepressionen durch Selektion gelingt bei beiden Wachstumsmerkmalen vollständig. Beim Körpergewicht am 21. Lebenstag ist jedoch đas zufällig sehr hohe Ausgangsniveau der Linie 2 im Verhältnis zu den anderen Linien zu berücksichtigen.

Tabelle 3

Mittelwerte des Merkmals Anzahl an Totgeburten im Wurf (TG) (Means of the stillbirths per litter)

\begin{tabular}{ccccc}
\hline Generation & $\mathrm{F}_{\mathrm{y}}$ & Linie 1 & Linie 2 & Linie 3 \\
\hline 0 & 0 & 0,07 & 0,05 & 0,08 \\
1 & 0,125 & 0,14 & 0,09 & 0,07 \\
2 & 0,218 & 0,12 & 0,09 & 0,08 \\
3 & 0,304 & 0,13 & 0,10 & 0,06 \\
4 & 0,380 & 0,15 & 0,10 & 0,07 \\
5 & 0,448 & 0,16 & 0,07 & 0,08 \\
6 & 0,508 & 0,09 & 0,08 & 0,06 \\
7 & 0,562 & 0,08 & 0,06 & 0,07 \\
8 & 0,610 & 0,08 & 0,06 & 0,08 \\
\hline
\end{tabular}

Tabelle 4

Mittelwerte des Merkmals Korpergewicht im Alter von 10 Tagen (KM10) (g) (Means of the body weight at the age of 10 days $(\mathrm{g}))$

\begin{tabular}{ccccc}
\hline Generation & $\mathrm{F}_{\mathrm{x}}$ & Linie 1 & Linie 2 & Linie 3 \\
\hline 0 & 0 & 12,40 & 12,60 & 12,20 \\
1 & 0,125 & 11,30 & 13,30 & 12,10 \\
2 & 0,218 & 10,90 & 12,60 & 12,40 \\
3 & 0,304 & 10,10 & 11,90 & 11,60 \\
4 & 0,380 & 9,90 & 12,40 & 11,70 \\
5 & 0,448 & 10,90 & 11,70 & 12,60 \\
6 & 0,508 & 10,60 & 10,90 & 12,20 \\
7 & 0,562 & 11,20 & 12,10 & 11,30 \\
8 & 0,610 & 11,30 & 12,80 & 11,90 \\
\hline
\end{tabular}

Tabelle 5

Mittelwerte des Merkmals Körpergewicht am 21. Lebenstag (KM21) (g) (Means of the body weight at the age of 21 days $(\mathrm{g}))$

\begin{tabular}{ccccc} 
21 days $(\mathrm{g})$ ) & $\mathrm{F}_{\mathrm{x}}$ & Linie I & Linie 2 & Linie 3 \\
\hline Generation & 0 & 24,20 & 29,40 & 25,10 \\
\hline 0 & 0,125 & 22,30 & 28,60 & 25,30 \\
1 & 0,218 & 22,00 & 27,60 & 24,90 \\
2 & 0,304 & 21,30 & 26,70 & 23,60 \\
3 & 0,380 & 20,80 & 28,30 & 25,20 \\
4 & 0,448 & 21,90 & 27,10 & 25,30 \\
5 & 0,508 & 22,00 & 25,20 & 26,60 \\
7 & 0,562 & 22,70 & 27,90 & 25,10 \\
8 & 0,610 & 20,30 & 27,60 & 25,00 \\
\hline
\end{tabular}

In der Tabelle 6 sind die linearen Regressionskoeffizienten des Inzuchtgrades auf die Fruchtbarkeits- und Wachstumsmerkmale als Schätzwerte für die Inzuchtdepression dargestellt. Dabei ist zwischen den absoluten Merkmalswerten und den Abweichungen von der Kontrolllinie unterschieden worden. Die Ergebnisse sind jedoch in beiden Merkmalsgruppen sehr ähnlich. Das trifft vor allem auf den Standardfehler der Regressionskoeffizienten, das Signifikanzniveau und die Korrelationskoeffizienten zwischen dem Inzuchtgrad und der Höhe der Merkmalswerte zu, die alle in gewissem Maße die Zuverißsssigkeit des geschätzten Regressionskoeffizienten widerspiegeln. 
Wie Tabelle 6 zu entnehmen ist, kann die Zuverlässigkeit durch die Verwendung der Abweichungen der Merkmale von der Kontrollinie nicht verbessert werden.

Ein signifikanter Einfluss der Inzucht auf die Merkmalshöhe kann nur bei der Wurfgröße und bei dem Körpergewicht am 21. Lebenstag beobachtet werden, wobei im ersteren Fall die absoluten Merkmalswerte und im letzteren die Abweichungen von der Kontrolllinie das Signifikanzniveau erreichten (Tab. 6).

Tabelle 6

Regressionen der durchschnittlichen Merkmalswerte auf den mittleren Inzuchtgrad (je 10\%) der Linie (Regression of the traits on the average degree of inbreeding of the line (per $10 \%)$ )

\begin{tabular}{lcccc}
\hline Merkmale & $\mathrm{b}$ & $\mathrm{s}_{\mathrm{b}}$ & $\alpha$ & $\mathrm{r}$ \\
\hline Absolute Merkmalswerte & & & & \\
Wurfgroße & $-0,524$ & 2,00 & 0,034 & $-0,76$ \\
Totgeburten & $-0,002$ & 0,06 & 0,78 & $-0,11$ \\
Korpergewicht 10. Tag & $-0,143$ & 1,24 & $0,, 28$ & $-0,40$ \\
Korpergewicht 21. Tag & $-0,336$ & 1,64 & 0,08 & $-0,61$ \\
Abweichungen von der Kontrolllinie & & & & $-0,55$ \\
Wurfgroße & $-0,420$ & 2,41 & 0,13 & $-0,07$ \\
Totgeburten & $-0,001$ & 0,07 & 0,87 & $-0,16$ \\
Korpergewicht 10. Tag & $-0,053$ & 1,26 & 0,69 & $-0,69$ \\
Korpergewicht 21. Tag & $-0,420$ & 1,69 & 0,04 &
\end{tabular}

4.

\section{Diskussion}

In der Literatur werden eine Vielzahl yon Selektionsexperimenten auf Fruchtbarkeit und Wachstum beschrieben. Genannt seien Arbeiten zur Langzeitselektion von BÜNGER u.a. (1983) und BÜNNGER (1993). Zum Einfluss der Inzucht gibt es dagegen weniger Informationen. RENNE u.a. (1997) und DIETL u.a. (2001) berichten über Langzeitselektionen auf Körpermasse und Fruchtbarkeit, bei welchen die in den Selektionslinien gegenüber den Kontrollpopulationen beobachteten Inzuchtdepressionen scheinbar nicht aufgehoben werden konnten.

Im vorliegenden Experiment wurde durch Halbgeschwisterpaarung in 8 Generationen ein Anstieg des mittleren Inzuchtkoeffizienten von 0 auf 0,61 erreicht. Trotz des rapiden Anstiegs des Inzuchtgrades halten sich die Inzuchtdepressionen in relativen Grenzen, Das trifft sowohl auf die Fruchtbarkeitsmerkmale als auch auf die Wachstumsmerkmale (Körpergewichte) zu, obwohi die Fruchtbarkeitsmerkmale auf Grund der niedrigen Heritabilität (BENIWAL u.a., 1992 a, b) im Allgemeinen noch stärker durch Inzuchtdepressionen gefährdet sind.

Die stärksten Depressionen traten bei allen untersuchten Merkmalen in den mittleren Generationen auf, in denen die durchschnittlichen Inzuchtkoeffizienten zwischen 30 und $50 \%$ lagen. Es kann vermutet werden, dass die natürliche Selektion in der Inzuchtlinie 1 den Depressionen in den letzten Generationen entgegengewirkt hat. Der offensichtlich nicht lineare Zusammenhang zwischen Inzuchtgrad und Inzuchtdepression liegt eventuell auch darin begründet, dass nicht nur Dominanzverluste als Ursache für die Inzuchtdepression verantwortlich sind, sondern auch epistatische Effekte eine Rolle spielen. Allerdings wäre dann eine degressive Abnahme der Merkmalswerte wahrscheinlicher. Die Situation ist schwer zu beurteilen, da der Homozygotiestatus beim Ausgang der Inzucht nicht bekannt ist. Es ist möglich, dass die nicht signifikanten linearen Zusammenhänge zwischen Inzuchtgrad und Merkmalshöhe durch den An- 
stieg der Generationsmittel der Merkmale in den letzten Generationen begründet liegen.

Bei allen untersuchten Merkmalen gelang es, in der Linie 2 durch Selektion die Inzuchtdepression in den Merkmalen zu kompensieren. Wegen der geringen Heritabilität ist das Ergebnis für die Fruchtbarkeitsmerkmale überraschend, da sie einerseits eine hohe Inzuchtdepression erwarten lassen und andererseits wegen der niedrigen Heritabilität nur einen begrenzten Zuchtfortschritt erreichen können. Außerdem ist die Intensität der Selektion geringer. Die offensichtlich problemlose Kompensation der Inzuchtdepression durch Selektion bei der Fruchtbarkeit ist ein ermutigendes Zeichen für die Anwendung der Inzucht zur Linienbildung innerhalb von Heterosiszuchtprogrammen.

\section{Literatur}

BENIWAL, B.K.; HASTINGS, M.; THOMPSON, R.; HILL, W.G.: Estimation of changes in genetic parameters in selected lines of mice using REML with an animal model. 1. Lean mass. Heredity 69 (1992 a), 352-360

BENIWAL, B.K.; HASTINGS, M.; THOMPSON, R.; HILL, W.G.;

Estimation of changes in genetic parameters in selected lines of mice using REML with an animal model. 2. Body weight, body composition and litter size. Heredity 69 (1992 b), 361-371

BÜNGER, L.; SCHÖLER, L.; KUPATZ, B.:

Zur Selektion auf Wachstum bei Modelltieren (Labormäusen). 2. Mitt.: Direkter Selektionserfolg. Arch. Tierz, Berlin 24 (1983), 281-293

BUNGER, L:

Long-term selection for body weight and fertility in mice with special consideration of a selection limit and it's causes, $44^{\text {th }}$ EAAP Meeting, Aarhus Ref. ID: 3250

DIETL, G.; RENNE, U.; LANGHAMMER, M.:

The simultaneus development of inbreeding, proportion of infertile matings and litter size in mouse lines long-term selected on high body weight under different selection procedures. $52^{\text {nd }}$ EAAP meeting, Budapest, August 26-29, 2001

PIRCHNER, F.; REINHARDT, F.; GOTTSCHALK, A.:

Die Verwendung von inzucht zur Steigerung des Selektionsfortschrittes. Züchtungskunde, Stuttgart 61 (1989) 1, 6-13

RENNE, U.; LANGHAMMER, M.; BÜNGER, L; DIETL, G.:

Effects of inbreeding on selection response in a mouse line tong-term selected for body weight. Arch. Tierz., Dummerstorf 40 (1997), Special Issue, 195

SMITH, C.; QUINTON, M.

The Effect of Selection in Sublines and Crossing on Genetic Response and Inbreeding. J. Anim. Sci. 71 (1993), 2631-2638

Eingegangen: 25.09 .2001

Akzeptiert: $17,10,2001$

Anschiriften der Verfasser

Dr. Agr. Ing. BASSAM SADALLA ISSA

Fakultăt für Landwirtschaft

der Universität Damaskus

P.O.Box 30621

Syrien

Prof, Dr. GERHARD SEELAND

Humboldt Universitat zu Berlin, Landwirtschaftlich-Gärtnerische Fakultăt

Institut fulr Nutztierwissenschaften

Fachgebiet Zuchtungsmethodik und Zuchtungsplanung

invalidenstr. 42

D.10115 Berlin 